\title{
Insulin enhances neointimal hyperplasia following arterial injury through the PI3K/Akt pathway in type 1 diabetic rats
}

\author{
JIN CAI ${ }^{1-3 *}$, HUAIMING WANG ${ }^{1,4 *}$, HONGFEI SANG ${ }^{1,5^{*}}$, RUIDONG YE $^{1}$, YUEZHOU CAO $^{1}$, MEI JING $^{2}$, \\ KETING LIU $^{1}$, YI XIE ${ }^{1}$, ZHONGMING QIU ${ }^{1,6}$, RUIBING GUO ${ }^{1}$, QIUSHI LV ${ }^{1}$ and XINFENG LIU ${ }^{1}$ \\ ${ }^{1}$ Department of Neurology, Jinling Hospital, Medical School of Nanjing University, Nanjing, Jiangsu 210002; \\ ${ }^{2}$ Department of Geriatrics, The 455th Hospital of Chinese People's Liberation Army; ${ }^{3}$ Department of Infectious Diseases, \\ Naval Medical Centre, Navy Medical University, Shanghai 200052; ${ }^{4}$ Department of Neurology, \\ The 80th Group Army Hospital of Chinese People's Liberation Army, Weifang, Shandong 261021; \\ ${ }^{5}$ Department of Neurology, Geriatrics Hospital of Hangzhou City, Hangzhou, Zhejiang 310022; ${ }^{6}$ Department of Neurology, \\ The 903rd Hospital of Chinese People's Liberation Army, Hangzhou, Zhejiang 310013, P.R. China
}

Received February 22, 2020; Accepted September 4, 2020

DOI: $10.3892 / \mathrm{mmr} .2020 .11628$

\begin{abstract}
Although insulin is known to affect neointimal hyperplasia via distinct signaling pathways, how neointimal hyperplasia is affected in insulin-deficient type 1 diabetes remains unknown. The aim of the current study was to investigate two major signaling branches of insulin action regulating neointimal hyperplasia following arterial injury in type 1 diabetes with or without exogenous insulin administration. Rats were treated with vehicle (control group), streptozotocin (STZ) alone (STZ group; uncontrolled type 1 diabetes) or STZ followed by insulin (STZ + I group; controlled type 1 diabetes). Subsequently, a type 1 diabetic rat model of carotid artery balloon injury was established. Following this, the intima-to-media area ratios were examined for evidence of neointimal hyperplasia in the carotid arteries of the rats by performing hematoxylin-eosin staining. Furthermore, the protein expression of extracellular signal-regulated kinase (ERK), phosphorylated (p-) ERK, protein kinase B (Akt) and p-Akt in the carotid arteries of the rats was determined via immunoblotting. Moreover, an in vitro model of type 1 diabetes was induced by incubation of primary vascular smooth muscle cells (VSMCs) with glucose and/or insulin.
\end{abstract}

Correspondence to: Dr Xinfeng Liu, Department of Neurology, Jinling Hospital, Medical School of Nanjing University, 305 East Zhongshan Road, Nanjing, Jiangsu 210002, P.R. China

E-mail: zfliu2@vip.163.com

${ }^{*}$ Contributed equally

Abbreviations: Akt, protein kinase B; ERK, extracellular signalregulated kinase; MAPK, mitogen-activated protein kinase; PI3K, phosphatidylinositol 3-kinase; VSMC, vascular smooth muscle cell

Key words: neointimal hyperplasia, type 1 diabetes, stenosis, proliferation
Cellular proliferation and signaling protein expression levels in VSMCs were determined by measuring the incorporation of tritiated thymidine and performing immunoblotting, respectively. The results demonstrated that compared with that in control rats, neointimal hyperplasia and expression of p-Akt in uncontrolled type 1 diabetic rats were significantly decreased. This decrease was recovered in controlled type 1 diabetes with insulin therapy. Furthermore, the difference in the expression of p-ERK between groups was not significant. Additionally, the results of the cell experiments were consistent with those from the animal studies. In conclusion, the preferential signaling along the phosphatidylinositol 3-kinase/Akt pathway of insulin action in response to insulin restoration may contribute to neointimal hyperplasia. The present study provides a novel approach for the further treatment of neointimal hyperplasia in type 1 diabetes.

\section{Introduction}

Diabetes is one of the most prevalent and costly chronic diseases worldwide, and has increased the morbidity of cardiovascular, cerebrovascular and peripheral arterial diseases $(1,2)$. The prevalence of adults with diabetes around the world in 2014 was $8.5 \%$ and in 2016, diabetes results in 1.6 million deaths $(3,4)$. Due to the increasingly aggressive and accelerated course of atherosclerosis in diabetes, patients have a greater probability of having strokes. In 2010, stroke was the second leading cause of death in patients $>60$ years old and the fifth leading cause of death in people aged 15-59 years worldwide $(5,6)$. As a result, diabetic patients with ischemic stroke undergo more revascularization procedures compared with the general population (7). Vascular interventions have several advantages, including microtrauma, short procedural duration and quick recovery, compared with carotid endarterectomy surgery, making them an essential treatment for carotid artery stenosis or occlusion (7). However, neointimal hyperplasia following the procedure, including balloon angioplasty and stenting is a common problem in patients with diabetes (8-10). 
Insulin affects neointimal hyperplasia via distinct signaling pathways (10-12). In vascular tissues, insulin stimulates two major signaling pathways: The phosphatidylinositol 3-kinase (PI3K)/protein kinase B (Akt) and mitogen-activated protein kinase (MAPK)/extracellular signal-regulated kinase (ERK) pathways (13). PI3K activation is essential for insulin-mediated glucose uptake, cell survival and nitric oxide (NO) production, while MAPK activation stimulates cellular proliferation and migration, and prothrombotic and proinflammatory responses (8). In the state of insulin resistance following balloon or stent injury, excess insulin stimulates signaling from the PI3K/Akt pathway to the MAPK/ERK pathway, and is involved in the endothelial production of NO and vascular smooth muscle cell (VSMC) proliferation and migration $(11,14)$. Furthermore, a higher ratio of phosphorylated (p-)ERK/total ERK to p-Akt/total Akt was associated with increased neointimal hyperplasia following vascular injury (11). Thus, it is evident that insulin is, at least in part, responsible for enhanced neointimal hyperplasia in insulin-resistant or type 2 diabetic models secondary to MAPK activation and/or PI3K impairment. However, given the different metabolic environments exhibited by patients with type 1 vs. type 2 diabetes, the detailed signaling pathways regulating neointimal hyperplasia in type 1 diabetes remain unclear.

Thus, we hypothesized that the PI3K/Akt or MAPK/ERK pathway regulated neointimal hyperplasia following arterial injury in type 1 diabetes, with or without insulin therapy. The preferential signaling along the PI3K/Akt pathway of insulin action in response to insulin deficiency may be involved. The current study performed in vitro cellular experiments and constructed an in vivo rat model of neointimal hyperplasia in type 1 diabetes, in which the roles of the PI3K/Akt and MAPK/ERK pathways were investigated. The present study provided a novel approach for the reduction of neointimal hyperplasia in type 1 diabetes.

\section{Materials and methods}

Animal model. The rats used in the current study were from the same strain as those used in our previous studies on type 1 diabetes $(15,16)$. A total of 30 male Sprague-Dawley (age, 11 weeks; weight, $\sim 300 \mathrm{~g}$ ) rats were maintained at the Animal Centre of Jinling Hospital (Nanjing, China). Rats were housed at room temperature with 12-h light/dark cycles, $60 \pm 5 \%$ relative humidity, and free access to food and water in a pathogen-free animal facility. Rats were randomly selected for a single intraperitoneal injection of streptozotocin (STZ; Sigma-Aldrich; Merck KGaA; $60 \mathrm{mg} / \mathrm{kg}$ dissolved in $\mathrm{pH} 4.2$ citrate buffer; $n=22$ ) or citrate buffer alone (control group; $\mathrm{n}=8)(15)$. A total of $19 \mathrm{STZ}$-treated rats (19/22) with a fasting blood glucose $>16.67 \mathrm{mmol} / 1(300 \mathrm{mg} / \mathrm{dl})$, which typically exhibits within 5 days of STZ injection, were considered as type 1 diabetic rats $(8,15)$. Rats $(n=3)$ with a low blood glucose $(<16.67 \mathrm{mmol} / \mathrm{l})$ within 7 days of STZ injection were excluded from the subsequent experiments. A subset of the type 1 diabetic rats $(9 / 19$; at random) received insulin glargine (Sanofi SA; 3 units; STZ + I group; controlled type 1 diabetes; $n=9$ ) daily via subcutaneous injection once hyperglycemia was detected. This treatment was continued daily for days prior to the establishment of the rat carotid injury model $(8,10)$. Insulin therapy in this group was continued for the remaining 2 weeks following surgery, with each rat receiving daily insulin administration for a total of 21 days. The other subset of the type 1 diabetic rats (10/19) without insulin administration were considered as uncontrolled type 1 diabetes (STZ group; $\mathrm{n}=10$ ).

Animal surgery. At $\sim$ day 14 following STZ injections, all 27 rats underwent surgery for the carotid artery balloon injury model. All animal procedures were performed according to the Guide for the Care and Use of Laboratory Animals published by the National Institutes of Health (NIH; publication no. 85-23; 1996) and approved by the Institutional Animal Care and Use Committee of Nanjing University (Nanjing, China). Rats were anesthetized with inhaled isoflurane (2-3\% induction; $0.5-1.5 \%$ maintenance) $(8,17)$. Atropine was administered subcutaneously $(0.1 \mathrm{mg} / \mathrm{kg})$ to decrease airway secretions. The neck was shaved and prepped with betadine and alcohol (75\%). Following a midline neck incision, the rat carotid artery balloon injury model was performed using a 2F Fogarty catheter (Edwards Lifesciences), as previously described $(8,12,18)$. Following injury and restoration of blood flow, the neck incision was closed. A total of 5 rats $(1$ in the control group and 2 in the STZ and STZ + I groups) died of cardiopulmonary arrest during surgery. Additionally, 4 rats (1 in the control group, 2 in the STZ group and 1 in the $\mathrm{STZ}+\mathrm{I}$ group) died of systemic embolism or serious infection during the follow-up period. A total of 18 surviving rats were euthanized at day 14 post-surgery by exposure to $\mathrm{CO}_{2}$ for 5 min (displacement rate, $20 \%$ of home cage volume/minute). Following this, cervical dislocation (rats weighing $<200 \mathrm{~g}$ ) or decapitation $(>200 \mathrm{~g})$ were performed under $\mathrm{CO}_{2}$ anesthesia. Presumed death was confirmed based on unambiguous signs of death, including cardiopulmonary arrest and/or fixed dilated pupils.

Morphometric analysis. Carotid arteries harvested at 2 weeks post-surgery were examined histologically for evidence of neointimal hyperplasia using routine hematoxylin-eosin staining. Briefly, the vessels were fixed with $4 \%$ paraformaldehyde at $4^{\circ} \mathrm{C}$ overnight. Then, the fixed vessels were embedded in paraffin, and 5- $\mu \mathrm{m}$ thick sections were cut and mounted on slides. The paraffin sections were stained with hematoxylin and eosin as previously described (15). Representative images of the aorta from the rats were observed under a light microscope (magnifications, $\mathrm{x} 10$ and $\mathrm{x} 20$ ). Both the intimal and medial areas were measured using ImageJ software (version 1.46r; National Institutes of Health) with uniform arbitrary units for subsequent calculation of the intima-to-media area (I/M) ratios.

Proliferation assay. VSMCs, isolated from rat carotid arteries as previously described (19), were characterized by smooth muscle cell morphology (multilayer sheets; 'hills and valleys') and smooth muscle $\alpha$-actin expression (19). A VSMC proliferation assay was conducted according to previous studies $(19,20)$. Briefly, primary VSMCs were plated in 12-well plates $\left(5 \times 10^{4}\right.$ cells/well $)$ and cultured at $37^{\circ} \mathrm{C}$ for $24 \mathrm{~h}$ in DMEM (Gibco; Thermo Fisher Scientific, Inc.) supplemented with 10\% FBS (Gibco; Thermo Fisher Scientific, Inc.). The cells 
Table I. Metabolic parameters of rats $(n=6)$.

\begin{tabular}{lccc}
\hline Characteristic & Control & STZ & STZ + I \\
\hline Initial age, weeks & 11 & 11 & 11 \\
Initial weight, g & $313 \pm 10$ & $315 \pm 8$ & $310 \pm 8$ \\
Final weight, g & $415 \pm 21$ & $289 \pm 9^{\mathrm{a}}$ & $413 \pm 16$ \\
Glucose, 72 h after STZ treatment, $\mathrm{mM}$ & $6.1 \pm 0.6$ & $20.8 \pm 3.0^{\mathrm{a}}$ & $20.4 \pm 3.3^{\mathrm{a}}$ \\
Glucose, 2 weeks after surgery, $\mathrm{mM}$ & $6.6 \pm 0.4$ & $21.1 \pm 2.2^{\mathrm{a}}$ & $7.3 \pm 0.5$ \\
Insulin, 2 weeks after surgery, $\mathrm{ng} / \mathrm{ml}$ & $4.32 \pm 0.47$ & $0.83 \pm 0.15^{\mathrm{a}}$ & $3.93 \pm 0.19$
\end{tabular}

Values are presented as mean \pm standard deviation $(n=6) .{ }^{\text {a }}<<0.05$ vs. control group. STZ, streptozotocin-injected rats without insulin therapy; $\mathrm{STZ}+\mathrm{I}$, streptozotocin-injected rats with insulin therapy.

were then exposed to serum-free media containing tritiated $\left({ }^{3} \mathrm{H}\right)$ thymidine $(1 \mu \mathrm{Ci} / \mathrm{ml}$; China Institute of Atomic Energy, $)$, glucose and/or bovine insulin for an additional $24 \mathrm{~h}$. Exposing VSMCs to normal or high glucose (5 or $25 \mathrm{mM}$ ) and/or normal insulin $(100 \mathrm{nM})$ concentrations for $24 \mathrm{~h}$ mimicked starved, normal, uncontrolled or controlled type 1 diabetes, respectively, as per previous studies $(8,21)$ (Table SI). $\left[{ }^{3} \mathrm{H}\right]$ thymidine incorporation into trichloroacetic acid-precipitated DNA was quantified by scintillation counting using a liquid scintillation counter (Beckman LS6500; Beckman Coulter, Inc.). Sorbitol $(25 \mathrm{mM})$ was used as an osmotic control for all experiments.

Immunoblotting. Immunoblotting was performed as previously described $(22,23)$. Briefly, total proteins were extracted from the carotid arteries or VSMCs treated without $\left[{ }^{3} \mathrm{H}\right.$ thymidine using RIPA Lysis Buffer (Beyotime Institute of Biotechnology). Protein concentrations were determined using the BCA Protein Assay kit (Thermo Fisher Scientific, Inc.). Samples containing $50 \mu \mathrm{g}$ protein or $10 \mu \mathrm{l}$ pre-stained molecular weight marker (cat. no. P0076; Beyotime Institute of Biotechnology) were separated via 10\% SDS-PAGE and electroblotted onto nitrocellulose membranes (Bio-Rad Laboratories). The membranes were blocked overnight with $5 \%$ nonfat dry milk in PBS-T [0.05\% Tween- 20 in $10 \mathrm{mmol} / 1$ $\mathrm{PBS}]$ at $4^{\circ} \mathrm{C}$ with constant shaking. The blots were probed with primary monoclonal rabbit anti-rat ERK (1:800; cat. no. 4695), Akt (1:800; cat. no. 4685), p-ERK (1:600; cat. no. 4370), p-Akt (1:600; cat. no. 4060) or GAPDH (1:1,000; cat. no. 5174) antibodies (all Cell Signaling Technology) overnight at $4^{\circ} \mathrm{C}$. Subsequently, the membranes were incubated with polyclonal IRDye ${ }^{\circledast} 800 \mathrm{CW}$-labeled goat anti-rabbit IgG secondary antibodies (1:10,000; cat. no. 102673-300; LI-COR Biosciences) for $1 \mathrm{~h}$ at room temperature in dark. Proteins were visualized using an infrared imaging system (LI-COR Biosciences). The density of each sample was calculated using Odyssey software (version 3.0; LI-COR Biosciences). The ratio of p-ERK/ERK to $\mathrm{p}-\mathrm{Akt} / \mathrm{Akt}$ in the carotid arteries was also calculated.

Blood chemistry assay. Blood glucose levels were screened on alternate days for 1 week following injection of STZ and twice weekly thereafter. Blood glucose was measured with a standardized, portable glucometer (Johnson \& Johnson) via puncture of the tail vein. Serum samples were collected from non-fasted animals at death and frozen at $-20^{\circ} \mathrm{C}$ until assay.
Insulin levels were determined by radioimmunoassay with an antibody (cat. no. SRI-13K; 1:1; Linco; EMD Millipore) made specifically against rat insulin. The rat insulin antibody had $100 \%$ cross-reactivity with human insulin.

Statistical analysis. Statistical analysis was performed using SPSS software (version 25.0; IBM Corp.). Data are expressed as the mean \pm standard deviation. Comparisons between groups were performed using one-way analysis of variance (ANOVA) followed by Duncan's and Tukey's ( $>3$ groups) post-hoc tests. $\mathrm{P}<0.05$ was considered to indicate a statistically significant difference.

\section{Results}

Metabolic parameters of SD rats. A total of 18, or two-thirds, of the rats survived until euthanasia, which was consistent with a published model of arterial injury in diabetic rats (10). The type 1 diabetic rats without exogenous insulin administration exhibited significant weight loss, increased blood glucose and decreased insulin. Blood glucose was also significantly increased at $72 \mathrm{~h}$ after STZ treatment in the STZ + I group compared with the control group. The sample size, age, weight, blood glucose and insulin levels in each group are presented in Table I.

Enhanced neointimal hyperplasia in STZ + I rats. The representative images of balloon-injured carotid artery in SD rats treated with controls, STZ and STZ + I stained with hematoxylin-eosin at day 14 post-surgery are presented in Fig. 1A. The upper and lower images were observed under a microscope at magnifications of $x 10$ and $x 20$, respectively, exhibiting the neointimal hyperplasia following arterial injury (Fig. 1A). The results demonstrated that the $\mathrm{I} / \mathrm{M}$ ratios were significantly decreased in the carotid arteries of the STZ rats compared with those in the controls (Fig. 1B). These levels were recovered and slightly increased in STZ + I rats (Fig. 1B); however, this increase was not significant.

Effect of insulin on Akt expression in balloon-injured carotid arteries. To elucidate the effect of insulin on the PI3K/Akt pathway in vivo, immunoblotting was used to measure the levels of p-Akt and total Akt in the carotid arteries of the rats (Fig. 2A). The results demonstrated that p-Akt/total Akt in the carotid arteries of STZ rats was significantly decreased 
A
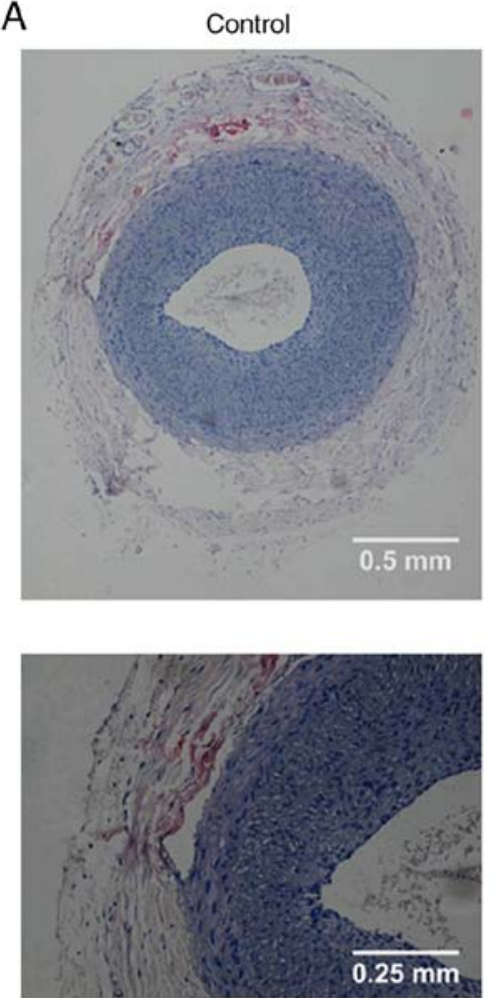

STZ
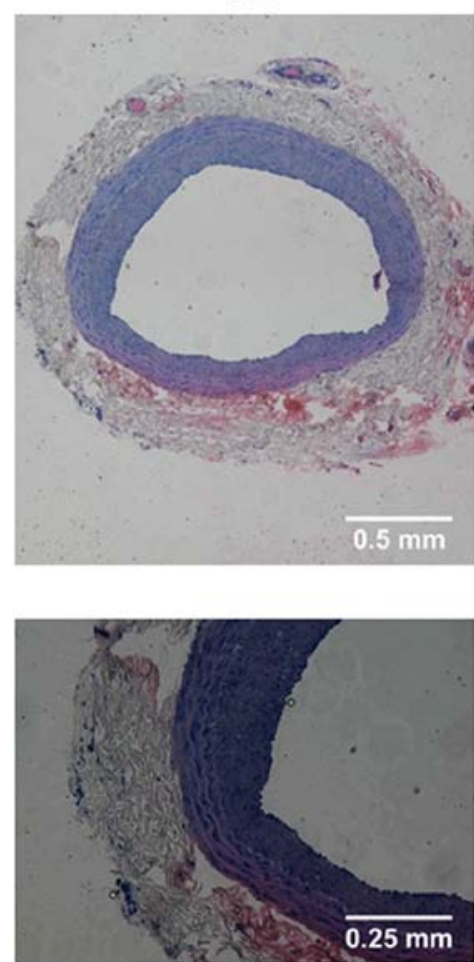

$\mathrm{STZ}+\mathrm{I}$
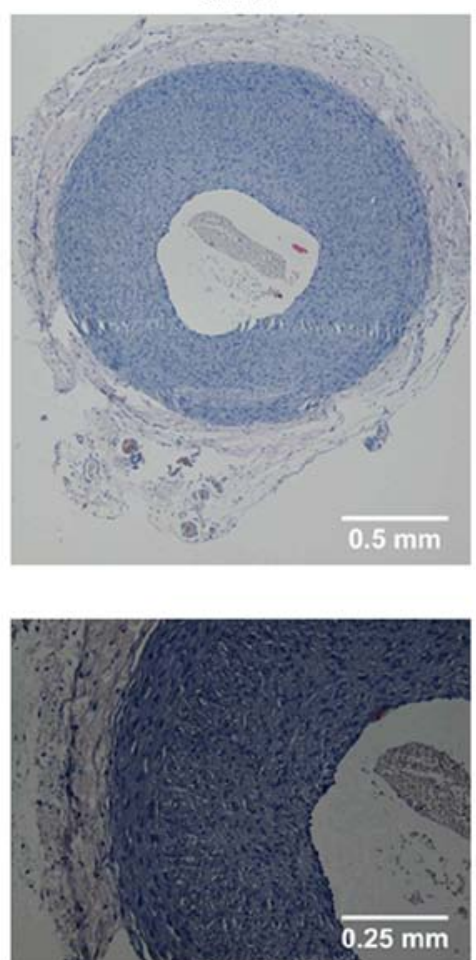

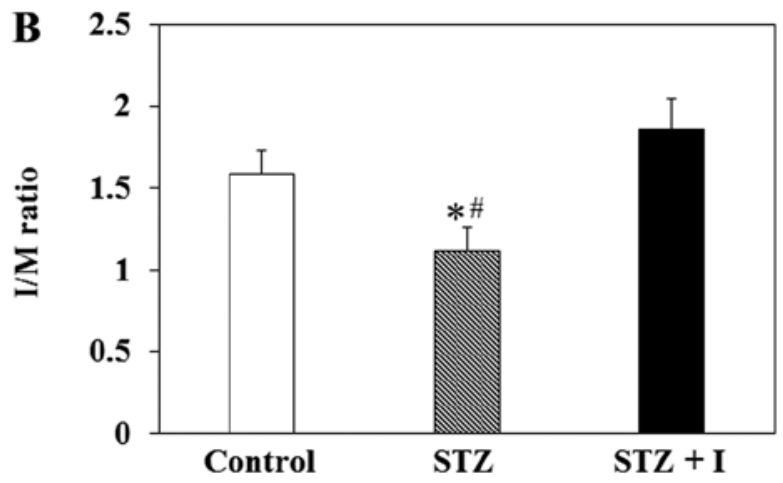

Figure 1. Effect of insulin on neointimal hyperplasia in balloon-injured carotid arteries. (A) Representative images of hematoxylin-eosin-stained carotid arteries from the rats treated with control, STZ or STZ followed by insulin at 14 days post-vascular balloon injury. The upper and lower images were observed under a microscope at magnifications of x10 and x20, respectively. (B) Bar graph demonstrating I/M ratios in the carotid arteries of rats. Values are presented as mean \pm standard deviation $(n=6)$. ${ }^{*} \mathrm{P}<0.05$ vs. control group; ${ }^{\#} \mathrm{P}<0.05$ vs. STZ + I group. STZ, streptozotocin; I, insulin; I/M, intima-to-media area.

compared with that in the control (Fig. 2B). This effect was ameliorated by insulin in the STZ + I group (Fig. 2B).

Effect of insulin on ERK expression in balloon-injured carotid arteries. To elucidate the influence of insulin on the MAPK/ERK pathway in vivo, immunoblotting was performed to measure the levels of p-ERK and total ERK in the carotid arteries of the rats (Fig. 3A). The results revealed that $\mathrm{p}-\mathrm{ERK} / \mathrm{ERK}$ in the carotid arteries of SD rats were not significantly different between groups (Fig. 3B).

Representative migration patterns of the pre-stained molecular weight marker and target proteins, including Akt, p-Akt, ERK, p-ERK and GAPDH, as determined by immunoblotting, are presented in Fig. S1.

Association between the effect of insulin and the ratio of $p$-ERK/ERK to $p$-Akt/Akt. To further clarify the effect of insulin on the MAPK/ERK and/or PI3K/Akt pathway, the ratio of $\mathrm{p}$-ERK/ERK to $\mathrm{p}-\mathrm{Akt} / \mathrm{Akt}$ in the carotid arteries was also calculated. The results demonstrated that the ratio of p-ERK/ERK to p-Akt/Akt was significantly increased in the STZ group compared with that in the control group (Fig. S2).

Cell proliferation and signaling proteins in VSMCs. To investigate VSMC proliferation in the state of uncontrolled or controlled type 1 diabetes, an in vitro assay was conducted using $\left[{ }^{3} \mathrm{H}\right]$ thymidine incorporation to serve as a surrogate for cell proliferation. Primary VSMCs exposed to the high glucose ( $25 \mathrm{mM}$ ) in the uncontrolled type 1 diabetes group demonstrated a significant reduction in proliferation compared with the low glucose $(5 \mathrm{mM})$ in the control or controlled type 1 diabetes groups (Fig. 4). Furthermore, the results demonstrated that the p-Akt/total Akt in the uncontrolled type 1 diabetes group was significantly decreased compared with the control or controlled 
A
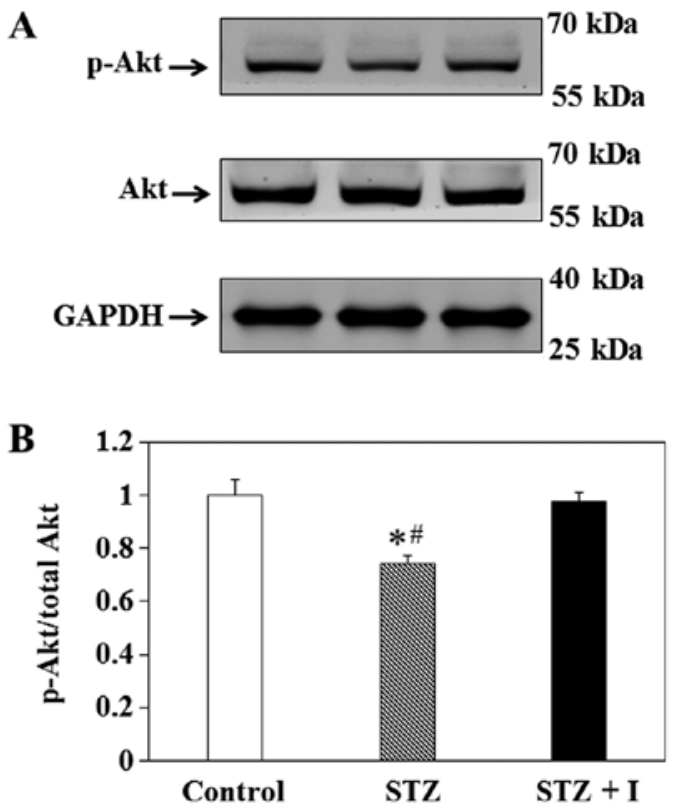

Figure 2. Akt expression in balloon-injured carotid arteries. (A) Immunoblotting with p-Akt (Ser473), total Akt and GAPDH in carotid arteries in the control, STZ and STZ + I rats 2 weeks post-surgery. Values are presented as relative to control. (B) Bar graph demonstrating p-Akt/total Akt expression in the carotid arteries of rats. Values are presented as the mean \pm standard deviation $(\mathrm{n}=6) .{ }^{*} \mathrm{P}<0.05$ vs. control group; ${ }^{*} \mathrm{P}<0.05$ vs. $\mathrm{STZ}+\mathrm{I}$ group. $\mathrm{p}-$, phosphorylated; STZ, streptozotocin; I, insulin; Akt, protein kinase B.
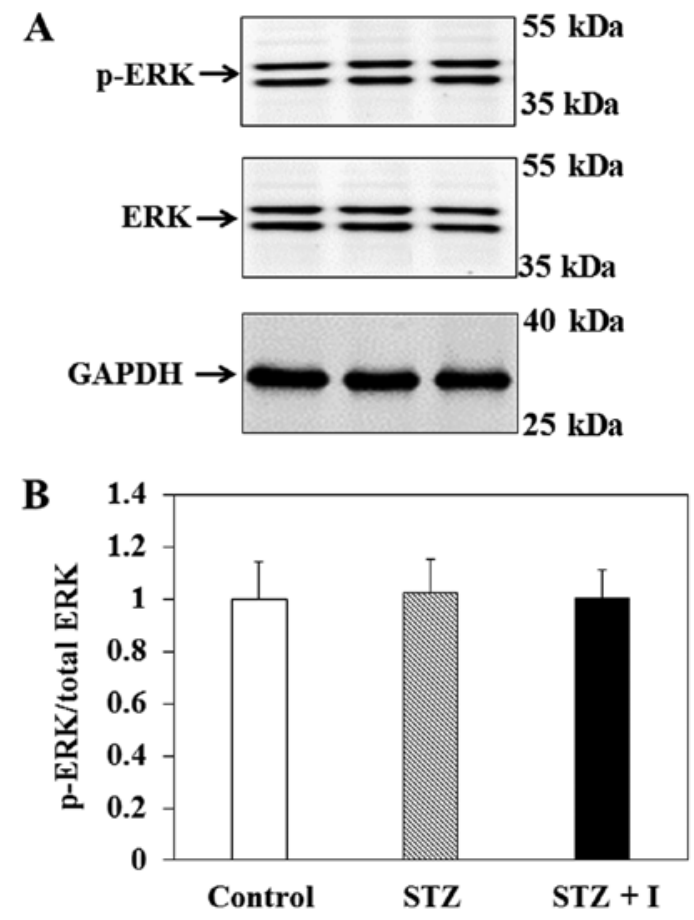

Figure 3. ERK expression in balloon-injured carotid artery. (A) Immunoblotting with p-ERK (Thr202/Tyr204), total ERK and GAPDH in the carotid arteries of control, STZ and STZ + I rats 2 weeks post-surgery. Values are presented as relative to control. (B) Bar graph demonstrating p-ERK/total ERK expression in the carotid arteries. Values are presented mean \pm standard deviation $(n=6)$. There was no significant difference between groups. p-, phosphorylated; ERK, extracellular signal-regulated kinase.

type 1 diabetes groups (Fig. 5). However, the p-ERK/total ERK in VSMCs was not significantly different between groups.

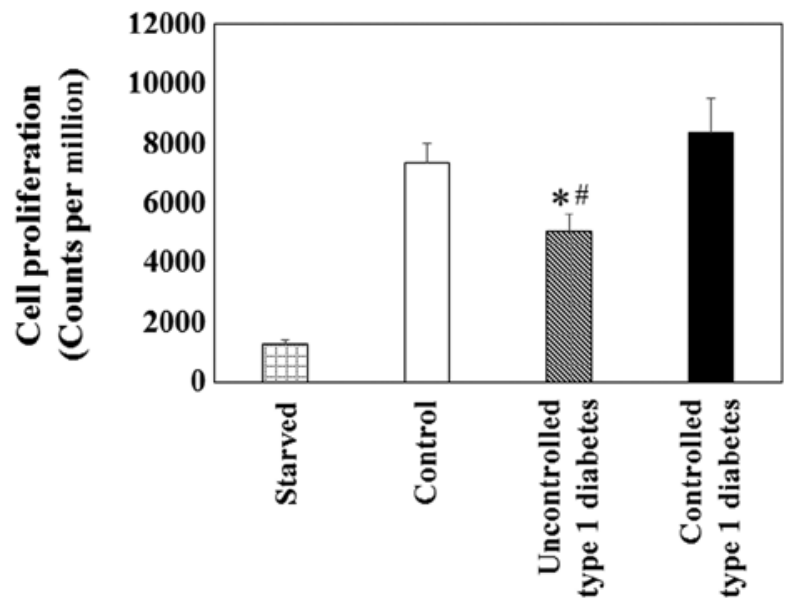

Figure 4. VSMC proliferation was assessed by $\left[{ }^{3} \mathrm{H}\right]$ thymidine incorporation. Primary VSMCs harvested from the carotid arteries rats were exposed to varying concentrations of glucose (5 or $25 \mathrm{mM})$ and/or insulin $(100 \mathrm{nM})$, which mimicked normal, uncontrolled or controlled type 1 diabetes. Values are presented as the mean $\pm \mathrm{SD}(\mathrm{n}=4)$. ${ }^{*} \mathrm{P}<0.05$ vs. control group; ${ }^{*} \mathrm{P}<0.05$ vs. controlled type 1 diabetes group. VSMC, vascular smooth muscle cell.

\section{Discussion}

The results of the current study demonstrated that, compared with controls, in uncontrolled type 1 diabetes both the neointimal hyperplasia and expression of p-Akt were significantly decreased, but were recovered following exogenous insulin administration in controlled type 1 diabetes. However, the difference in the expression of p-ERK was not significant between groups. Furthermore, the cellular results of the in vitro experiments were consistent with those from the in vitro animal experiments. These findings indicated that high glucose may inhibit VSMC proliferation by inhibiting the PI3K/Akt signaling pathway in type 1 diabetes with low insulin levels, which can be improved by exogenous insulin supplementation.

Consistent with previous studies, the results of the current study demonstrated that PI3K/Akt signaling was impaired in uncontrolled type 1 diabetes, compared with controls, most likely due to low insulin and subsequent high glucose $(11,14,24)$. The present study indicated that once the insulin and glucose levels normalized, the impairment was recovered. Unexpectedly, neointimal hyperplasia was slightly increased, but not significant, in controlled type 1 diabetes compared with the controls. A possible explanation is that Akt activation by abundant insulin in VSMCs may be necessary for maintaining a quiescent, fully differentiated phenotype rather than a migratory, proliferative one $(14,21)$. Furthermore, endothelial production of NO induced by Akt activation may result in vascular protective effects $(25,26)$. However, in the setting of insulin resistance, the balance that normally regulates VSMC proliferation/migration is disrupted by differentially shunted signaling from Akt to ERK activation (11). Furthermore, Akt inhibition coupled with excess insulin may lead to endothelial reduced production of $\mathrm{NO}$ and increased expression of cellular adhesion molecules, such as CD11a or ICAM-1, with increased monocyte rolling and arrest (27).

Additionally, the results of the present study reported that MAPK/ERK signaling was not significantly different in uncontrolled or controlled type 1 diabetes, with low or normal 

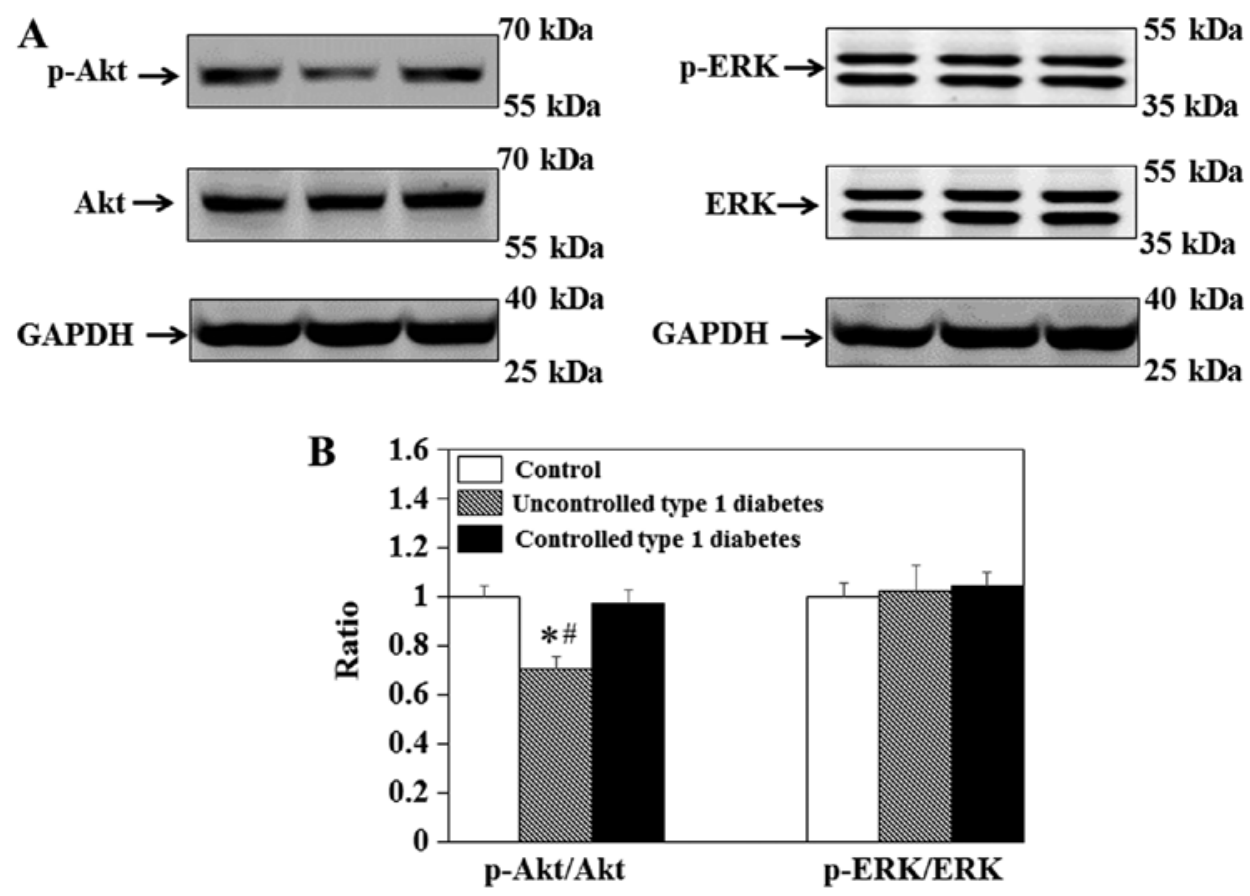

Figure 5. Akt and ERK expression in VSMCs. (A) Immunoblotting of phosphorylated p-Akt (Ser473), total Akt, p-ERK (Thr202/Tyr204), total ERK and GAPDH in VSMCs treated with varying concentrations of glucose $(5$ or $25 \mathrm{mM})$ and/or insulin $(100 \mathrm{nM})$. Data are presented as relative to control. (B) Bar graph demonstrated the ratio of $\mathrm{p}-\mathrm{Akt} / \mathrm{Akt}$ and $\mathrm{p}-\mathrm{ERK} / \mathrm{ERK}$ expression in VSMCs. Values are presented as mean \pm standard deviation ( $\mathrm{n}=4)$. " $\mathrm{P}<0.05 \mathrm{vs}$. control group; ${ }^{\#} \mathrm{P}<0.05$ vs. controlled type 1 diabetes group. VSMCs, vascular smooth muscle cells; p-, phosphorylated; Akt, protein kinase B; ERK, extracellular signal-regulated kinase.

insulin levels, respectively. However, as described previously, in the setting of insulin resistance, the MAPK/ERK signaling pathway may be overactive, promoting proliferation and migration; therefore, a higher ratio of p-ERK/ERK to p-Akt/Akt was associated with increased neointimal hyperplasia following vascular injury $(8,11,14)$. The results of the current study demonstrated that the increased ratio of $p-E R K / E R K$ to p-Akt/Akt did not produce increased neointimal hyperplasia following vascular injury in the setting of uncontrolled type 1 diabetes with low insulin. Nevertheless, the increased ratio of $\mathrm{p}$-ERK/ERK to $\mathrm{p}$-Akt/Akt in controlled type 1 diabetic rats compared with control rats with normal insulin levels may, at least in part, be associated with enhanced neointimal hyperplasia following vascular injury, which is consistent with previously published studies $(10,11,14,28)$.

The current study had limitations. Data were both similar to $(8,28)$ and different from $(10,29)$ published studies, indicating that glucose can stimulate and inhibit VSMC proliferation $(8,10,28,29)$. Regardless, the current study also observed a direct association between in vitro and in vivo experiments with regard to VSMC proliferation and neointimal hyperplasia in type 1 diabetes with insulin therapy. Notably, the results reported that PI3K/Akt signaling served a further important role in VSMC proliferation in the type 1 diabetic model with different insulin levels. Additionally, the insulin levels in type 1 diabetic rats indicated the damage to the pancreatic tissue induced by STZ, irrespective of insulin administration. However, the pancreatic tissue should be examined to confirm $\beta$ cell damage in future studies.

The preferential signaling along the PI3K/Akt pathway of insulin action in response to insulin restoration may contribute to neointimal hyperplasia. The present study provided a novel method for further treatment of atherosclerosis in type 1 diabetes.

\section{Acknowledgements}

Not applicable.

\section{Funding}

The current study was supported by the National Natural Science Foundation of China (grant nos. 81400332, 81571148 and 81701229).

\section{Availability of data and materials}

The datasets used and/or analyzed during the current study are available from the corresponding author on reasonable request.

\section{Authors' contributions}

JC conceived the current study, analyzed data and wrote the manuscript. HW and HS acquired and analyzed data. RY, YC, $\mathrm{KL}$ and ZQ performed the experiments. MJ, YX, RG and QL analyzed data and revised the manuscript. XL conceived the current study and revised the manuscript. All authors read and approved the final manuscript.

\section{Ethics approval and consent to participate}

All animal procedures were performed according the Guide for the Care and Use of Laboratory Animals published by 
the NIH (publication no. 85-23; 1996) and approved by the Institutional Animal Care and Use Committee of Nanjing University, Nanjing, China.

\section{Patient consent for publication}

Not applicable.

\section{Competing interests}

The authors declare that they have no competing interests.

\section{References}

1. Narayan KM, Boyle JP, Thompson TJ, Sorensen SW and Williamson DF: Lifetime risk for diabetes mellitus in the United States. JAMA 290: 1884-1890, 2003.

2. Beckman JA, Creager MA and Libby P: Diabetes and atherosclerosis: Epidemiology, pathophysiology, and management. JAMA 287: 2570-2581, 2002.

3. Liu X, Yu C, Wang Y, Bi Y, Liu Y and Zhang ZJ: Trends in the incidence and mortality of diabetes in China from 1990 to 2017 A joinpoint and age-period-cohort analysis. Int J Environ Res Public Health 16: 158, 2019.

4. NCD Risk Factor Collaboration (NCD-RisC): Worldwide trends in diabetes since 1980: A pooled analysis of 751 population-based studies with 4.4 million participants. Lancet 387: 1513-1530, 2016

5. Yang G, Wang Y, Zeng Y, Gao GF, Liang X, Zhou M, Wan X, Yu S, Jiang Y, Naghavi M, et al: Rapid health transition in China, 1990-2010: Findings from the global burden of disease study 2010. Lancet 381: 1987-2015, 2013.

6. Liu L, Wang D, Wong KS and Wang Y: Stroke and stroke care in China: Huge burden, significant workload, and a national priority. Stroke 42: 3651-3654, 2011.

7. Sakai N and Sakai C: Current indication of carotid diseases, CEA vs CAS. Nihon Geka Gakkai Zasshi 111: 75-78, 2010 (In Japanese)

8. Varu VN, Ahanchi SS, Hogg ME, Bhikhapurwala HA, Chen A, Popowich DA, Vavra AK, Martinez J, Jiang Q, Saavedra JE, et al: Insulin enhances the effect of nitric oxide at inhibiting neointimal hyperplasia in a rat model of type 1 diabetes. Am J Physiol Heart Circ Physiol 299: H772-H779, 2010.

9. Cao Y, Wang S, Sun W, Dai Q, Li W, Cai J, Fan X, Zhu W, Xiong Y, Han Y, et al: Prediction of favorable outcome by percent improvement in patients with acute ischemic stroke treated with endovascular stent thrombectomy. J Clin Neurosci 38: 100-105, 2017.

10. Park SH, Marso SP, Zhou Z, Foroudi F, Topol EJ and Lincoff AM: Neointimal hyperplasia after arterial injury is increased in a rat model of non-insulin-dependent diabetes mellitus. Circulation 104: 815-819, 2001.

11. Jonas M, Edelman ER, Groothuis A, Baker AB, Seifert P and Rogers C: Vascular neointimal formation and signaling pathway activation in response to stent injury in insulin-resistant and diabetic animals. Circ Res 97: 725-733, 2005.

12. Ahanchi SS, Varu VN, Tsihlis ND, Martinez J, Pearce CG, Kapadia MR, Jiang Q, Saavedra JE, Keefer LK, Hrabie JA and Kibbe MR: Heightened efficacy of nitric oxide-based therapies in type II diabetes mellitus and metabolic syndrome. Am J Physiol Heart Circ Physiol 295: H2388-H2398, 2008.

13. White MF: Insulin signaling in health and disease. Science 302 : $1710-1711,2003$

14. Wang CC, Gurevich I and Draznin B: Insulin affects vascular smooth muscle cell phenotype and migration via distinct signaling pathways. Diabetes 52: 2562-2569, 2003.
15. Sang H, Qiu Z, Cai J, Lan W, Yu L, Zhang H, Li M, Xie Y, Guo R, Ye R, et al: Early increased bradykinin 1 receptor contributes to hemorrhagic transformation after ischemic stroke in type 1 diabetic rats. Transl Stroke Res 8: 597-611, 2017.

16. Sang H, Liu L, Wang L, Qiu Z, Li M, Yu L, Zhang H, Shi R, Yu S, Guo R, et al: Opposite roles of bradykinin B1 and B2 receptors during cerebral ischaemia-reperfusion injury in experimental diabetic rats. Eur J Neurosci 43: 53-65, 2016.

17. Kenny JD, Chemali JJ, Cotten JF, Van Dort CJ, Kim SE, Ba D, Taylor NE, Brown EN and Solt K: Physostigmine and methylphenidate induce distinct arousal states during isoflurane general anesthesia in rats. Anesth Analg 123: 1210-1219, 2016.

18. Shears LL II, Kibbe MR, Murdock AD, Billiar TR, Lizonova A, Watkins SC and Tzeng E: Efficient inhibition of intimal hyperplasia by adenovirus-mediated inducible nitric oxide synthase gene transfer to rats and pigs in vivo. J Am Coll Surg 187: 295-306, 1998.

19. Li Z, Yu C, Han Y, Ren H, Shi W, Fu C, He D, Huang L, Yang C, Wang X, et al: Inhibitory effect of D1-like and D3 dopamine receptors on norepinephrine-induced proliferation in vascular smooth muscle cells. Am J Physiol Heart Circ Physiol 294: H2761-H2768, 2008.

20. Zhou Y, Shi W, Luo H, Yue R, Wang Z, Wang W, Liu L, Wang WE, Wang H and Zeng C: Inhibitory effect of D1-like dopamine receptors on neuropeptide Y-induced proliferation in vascular smooth muscle cells. Hypertens Res 38: 807-812, 2015.

21. Torella D, Iaconetti C, Tarallo R, Marino F, Giurato G, Veneziano C, Aquila I, Scalise M, Mancuso T, Cianflone E, et al: miRNA regulation of the hyperproliferative phenotype of vascular smooth muscle cells in diabetes. Diabetes 67: 2554-2568, 2018.

22. Cai J, Han Y, Ren H, Chen C, He D, Zhou L, Eisner GM, Asico LD, Jose PA and Zeng C: Extracellular vesicle-mediated transfer of donor genomic DNA to recipient cells is a novel mechanism for genetic influence between cells. J Mol Cell Biol 5: 227-238, 2013

23. Wu G, Cai J, Han Y, Chen J, Huang ZP, Chen C, Cai Y, Huang H, Yang Y, Liu Y, et al: LincRNA-p21 regulates neointima formation, vascular smooth muscle cell proliferation, apoptosis, and atherosclerosis by enhancing p53 activity. Circulation 130 : 1452-1465, 2014.

24. Gong Z, Han Y, Wu L, Xia T, Ren H, Yang D, Gu D, Wang H, $\mathrm{Hu}$ C, He D, et al: Translocator protein $18 \mathrm{kDa}$ ligand alleviates neointimal hyperplasia in the diabetic rat artery injury model via activating PKG. Life Sci 221: 72-82, 2019.

25. Zeng G, Nystrom FH, Ravichandran LV, Cong LN, Kirby M, Mostowski $\mathrm{H}$ and Quon MJ: Roles for insulin receptor, PI3-kinase, and Akt in insulin-signaling pathways related to production of nitric oxide in human vascular endothelial cells. Circulation 101: 1539-1545, 2000.

26. Kuboki K, Jiang ZY, Takahara N, Ha SW, Igarashi M, Yamauchi T, Feener EP, Herbert TP, Rhodes CJ and King GL: Regulation of endothelial constitutive nitric oxide synthase gene expression in endothelial cells and in vivo: A specific vascular action of insulin. Circulation 101: 676-681, 2000.

27. Montagnani M,Chen H,Barr VA and Quon MJ: Insulin-stimulated activation of eNOS is independent of $\mathrm{Ca}^{2+}$ but requires phosphorylation by Akt at Ser(1179). J Biol Chem 276: 30392-30398, 2001.

28. Indolfi C, Torella D, Cavuto L, Davalli AM, Coppola C, Esposito G, Carriero MV, Rapacciuolo A, Di Lorenzo E, Stabile E, et al: Effects of balloon injury on neointimal hyperplasia in streptozotocin-induced diabetes and in hyperinsulinemic nondiabetic pancreatic islet-transplanted rats. Circulation 103: 2980-2986, 2001.

29. Zhuang D, Pu Q, Ceacareanu B, Chang Y, Dixit M and Hassid A: Chronic insulin treatment amplifies PDGF-induced motility in differentiated aortic smooth muscle cells by suppressing the expression and function of PTP1B. Am J Physiol Heart Circ Physiol 295: H163-H173, 2008. 